\title{
Wavelength Converter Sharing in Asynchronous Optical Packet/Burst Switching: An Exact Blocking Analysis for Markovian Arrivals
}

\author{
Nail Akar, Member, IEEE, Ezhan Karasan, Member, IEEE, and Kaan Dogan, Student Member, IEEE
}

\begin{abstract}
In this paper, we study the blocking probabilities in a wavelength division multiplexing-based asynchronous bufferless optical packet/burst switch equipped with a bank of tuneable wavelength converters dedicated to each output fiber line. Wavelength converter sharing, also referred to as partial wavelength conversion, corresponds to the case of a number of converters shared amongst a larger number of wavelength channels. In this study, we present a probabilistic framework for exactly calculating the packet blocking probabilities for optical packet/burst switching systems utilizing wavelength converter sharing. In our model, packet arrivals at the optical switch are first assumed to be Poisson and later generalized to the more general Markovian arrival process to cope with very general traffic patterns whereas packet lengths are assumed to be exponentially distributed. As opposed to the existing literature based on approximations and/or simulations, we formulate the problem as one of finding the steady-state solution of a continuous-time Markov chain with a block tridiagonal infinitesimal generator. To find such solutions, we propose a numerically efficient and stable algorithm based on block tridiagonal $\mathrm{LU}$ factorizations. We show that exact blocking probabilities can be efficiently calculated even for very large systems and rare blocking probabilities, e.g., systems with 256 wavelengths per fiber and blocking probabilities in the order of $10^{-40}$. Relying on the stability and speed of the proposed algorithm, we also provide a means of provisioning wavelength channels and converters in optical packet/burst switching systems.
\end{abstract}

Index Terms-Optical packet switching, optical burst switching, wavelength conversion, converter sharing, block-tridiagonal LU factorization, Markovian arrival process.

\section{INTRODUCTION}

D RAMATIC growth in Internet traffic demands has led to the use of Wavelength Division Multiplexing (WDM) systems which multiplex wavelengths of different frequencies onto a single fiber. This multiplexing operation creates multiple channels on the same fiber each carrying a different signal. Today's long-haul WDM systems support up to 160 channels each having a capacity of $10 \mathrm{~Gb} / \mathrm{s}$ with an overall capacity of $1.6 \mathrm{~Tb} / \mathrm{s}$ on a single fiber. On the other hand, client requirements are very diverse in terms of required capacity, connection utilization (continuous or bursty), connection duration, connection set-up times, etc. Using circuit switching at wavelength or subwavelength levels, today's wavelengthrouted WDM networks route connection requests through the

Manuscript received December 5, 2005; revised September 28, 2006. This work is supported in part by The Science and Research Council of Turkey (Tübitak) under projects EEEAG-106E046, EEEAG-104E047, and by the Commission of the European Community IST-FP6 e-Photon/ONe project.

The authors are with the Electrical and Electronics Engineering Department, Bilkent University, Ankara, Turkey.

Digital Object Identifier 10.1109/JSAC-OCN.2006.024305. optical network to account for diversified capacity needs of users. In this switching paradigm, connection durations and set-up times tend to be very long and dynamic bandwidth sharing across clients is minimal which gives rise to inefficient bandwidth use especially for bursty data traffic.

To cope with bursty traffic and for more efficient utilization of the fiber capacity, two new packet-based optical switching paradigms have been introduced: Optical Packet Switching (OPS) [1],[2] and Optical Burst Switching (OBS) [3],[4]. OPS requires line-rate header parsing and is thus viewed as a longer term solution due to the current technological limitations in packet header processing [2]. OBS, on the other hand, eliminates the need for header parsing by segregating the control and data planes. In OBS, the reservation request for a burst is signalled out of band by the use of a burst control packet which is processed in the electronic domain whereas the burst itself is transported end-to-end completely in the optical domain. When the control packet arrives at an optical burst switch towards its destination, the switch is configured for the corresponding data burst which would arrive after an offset time. Under the just enough time (JET) reservation protocol, allocated resources are released as soon as the burst is transmitted by the switch [3]. Although the control planes of OPS and OBS are different, both of them have similar data planes. Throughout this paper, the terms "(optical) packet" and "(optical) packet switching" refer to a packet/burst and the data plane of OPS/OBS, respectively, since the analysis we pursue involves only the data plane of optical packet/burst switching.

We consider the online blocking model described in [5] in which lightpaths are set up and torn down at the packet level. In synchronous (i.e., time-slotted) optical packet switching networks, packet lengths are fixed and packets are assumed to arrive at slot boundaries [6]. In asynchronous (i.e., unslotted) networks, packets can have variable sizes and are not aligned before they enter the optical switch [6]. Slotted switching systems generally lead to a better performance than their unslotted counterparts since the arrival behavior of the incoming packets to the switch is regulated in the former type leading to fewer contentions [7]. Moreover, the packet loss rate at a bufferless synchronous optical switch does not depend on the autocorrelation structure of the incoming traffic thereby making combinatorial analysis techniques possible. On the down side, it is more costly to implement synchronous optical packet switching nodes with high data rates since it requires the synchronization of all incoming packets in the 
optical domain. On the other hand, the node architecture for asynchronous networks is simpler because there is no need for the synchronization stage [6]. Furthermore, the asynchronous operation is a more natural fit for switching IP packets. For these reasons, it is worthwhile to consider asynchronous operation with variable-sized packets and our focus in this study is on asynchronous packet switching.

One of the major issues in optical packet switching networks is contention which arises as a result of two or more incoming packets contending for the same output wavelength. Contention is resolved either in wavelength domain by wavelength converters, in time domain by Fiber Delay Lines (FDL), or in space domain by deflection routing [3]. If contention cannot be resolved by any one of the proposed techniques, then one or more contending packets will be blocked. Calculating the packet blocking probabilities is crucial in evaluating the performance of optical packet switches with a certain contention resolution capability. In this paper, we study the packet blocking probabilities for the full-range "wavelength conversion" approach in which Tunable Wavelength Converters (TWC) are used for switching optical packets from any input wavelength onto any output wavelength for contention resolution. In Full Wavelength Conversion (FWC), a packet arriving at a certain wavelength channel can be switched onto any other idle wavelength channel towards its destination. FWC reduces packet blocking probabilities significantly compared with the case of No Wavelength Conversion (NWC) [8],[9],[10]. However implementing all-optical FWC is very costly.

Converter sharing or equivalently Partial Wavelength Conversion (PWC) is proposed as a cost-conscious alternative to FWC [11]. In PWC, there is a limited number of TWCs, and consequently some optical packets cannot be switched towards their destination, i.e., blocked, when all converters are busy despite the availability of free wavelength channels on the output fiber. In PWC, TWCs may be configured as a single converter pool for converter sharing across all fiber lines, which is referred to as the Share-Per-Node (SPN) architecture [7]. An alternative architecture allows separate TWC banks per output fiber and the corresponding solution is called the SharePer-Line (SPL) architecture. Although the SPN architecture leads to a better performance, the complexity of the switching matrix is lower in the SPL architecture [7]. Our focus in this study will be on SPL type converter sharing. We also note that a Share-Per-Input-Line (SPIL) architecture is proposed in [12] where a bank of TWCs is shared for all packets arriving on the same input fiber but SPIL is outside the scope of the current paper.

Another issue regarding wavelength conversion is whether there is a specified range of wavelengths that a given wavelength can be converted to. Such a converter is said to be limited-range. If there is no tuning range limit then the converter is called full-range. The focus of the current paper is on the blocking analysis of asynchronous optical packet switches using simpler-to-implement SPL-type converter sharing and with full-range TWCs. This model is depicted in Fig. 1 which presents a diagram of an optical packet switch with an internally non-blocking switch fabric and which has $N$ input and output fiber lines, $K$ wavelength channels per fiber, and a

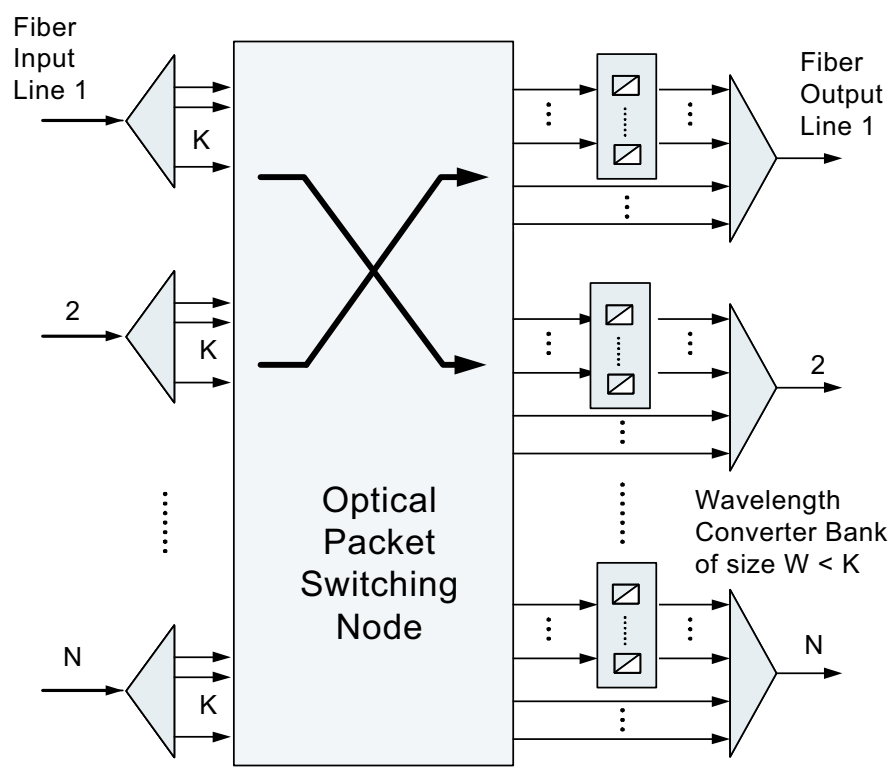

Fig. 1. The general architecture of an optical packet switching node with $N$ fiber I/O lines, $K$ wavelength channels on each fiber line, and a bank of wavelength converters of size $W$ shared per output line.

wavelength converter bank of size $W<K$ dedicated to each output fiber. We note that we will also study the limiting cases $W=0$ and $W=K$ which correspond to NWC and FWC architectures, respectively.

For the general PWC case, there is no closed form expression for the blocking probability and analytical results are also rare [13]. To the best of our knowledge, a numerical CTMC (Continuous Time Markov Chain) based algorithm for PWC in asynchronous SPL architectures is first proposed in [14]. Recently, a similar CTMC-based analysis is also proposed in [15] for the SPL case with Poisson packet arrivals and exponentially distributed packet lengths, and an approximate analysis is proposed for the SPN case. Although the analysis of [15] for the SPL case is similar to that of [14], the block structure of the CTMC is not exploited and the authors report problems in large systems (e.g., $K=64$ ) and for rare blocking probabilities. In [13], a fixed-point iteration-based approximate method for PWC for the SPN architecture is mentioned but not detailed. The work in [16] is simulation-based and attempts to find the number of wavelength converters required to attain a desired GoS (Grade of Service) in terms of blocking rates. In [7], simulations and an approximate analysis are provided for the same problem for the asynchronous case. Poisson and non-Poisson dynamic traffic patterns are simulated in [16] and [17] to show the impact of the packet arrival process on throughput. Simulation is generally a very useful tool since it can be applied to a wide range of models but i) simulations typically suffer from long run-times, ii) studying rare blocking probabilities may not be feasible, iii) it is computationally costly to use simulations in scenarios that might require iterations (e.g., analysis of networks, design and dimensioning problems, etc.).

In this paper, first we exactly calculate the packet blocking probabilities for a bufferless asynchronous optical packet switch with SPL-type converter sharing using the usual Poisson arrival and exponential packet length assumptions. As 
opposed to existing literature based on approximations and/or simulations and following [14], we formulate the problem as one of finding the steady-state solution of a Continuous-Time Markov Chain (CTMC) with a block tridiagonal infinitesimal generator. For Poisson arrivals, we propose a numerically efficient and stable solution technique based on block tridiagonal LU factorizations. We show that blocking probabilities can be found exactly and efficiently even for very large systems and rare blocking probabilities. Using the proposed solution method, we also present a design procedure for provisioning wavelength channels and converters in the case of Poisson packet traffic.

We also generalize our results to more general packet arrivals that are modelled with the Markovian Arrival Process (MAP) that was first introduced in [18]; also see [19],[20],[21]. The MAP is a versatile process that allows correlation in packet interarrival times. Moreover, MAPs have a significant property that they are dense in the set of all stationary point processes. Special cases of the MAP are widely used in the literature for a variety of scenarios; the Interrupted Poisson Process (IPP) to approximate overflow traffic [22], a Phase Type (PH)-type process for fitting long-tailed data [23], a twostate Markov Modulated Poisson Process (MMPP) to describe the superposition of a number of on-off packet sources [24], more general MMPPs to model correlated aggregate Internet traffic [25],[26],[27]. There are several possibilities to use the MAP as a traffic modeling tool in optical packet switching networks; i) measuring the optical packet traffic directly and fitting a MAP to data, ii) using the existing aggregate Markovian Internet traffic models together with the packetizer at the edge of the optical domain to build MAP-based traffic models for the optical core to accurately capture the bursty nature of the traffic, iii) using a MAP for representing overflow traffic to study the performance of optical packet switching networks. The use of MAP in optical packet switching networks is relatively new: for example [28] uses a two-state MMPP for burst generation in an OBS network and [29] uses a twostate hyperexponential arrival process (a subcase of MAP) to be fed to an OPS network. In this paper, we use the same technique used for Poisson arrivals for SPL analysis this time for the case of MAP traffic and derive an expression for the packet blocking probability which gives us a way of studying the effect of traffic characteristics on packet switch performance with PWC. In particular, we study the impact of autocorrelation in the optical packet arrival process on packet blocking performance by using a MAP with a fixed marginal but a varying autocorrelation function.

The remainder of this paper is organized as follows. Section II describes the model with Poisson packet arrivals as well as the solution procedure. The extension to MAP arrivals is studied in Section III. Section IV is devoted to numerical examples. We conclude in the final section.

\section{AnAlysis of SPL ARCHItecture FOR Poisson TRAFFIC CASE}

We study the asynchronous optical packet switch in Fig. 1 which has $N$ input and output fiber lines and $K$ wavelength channels per fiber. We assume a wavelength converter bank of size $0 \leq W \leq K$ dedicated to each output fiber. Optical packets destined to a particular output fiber line arrive at the switch from one of the $N$ input fibers. We assume that the wavelength channel a packet arrives on is uniformly distributed on $(1, K)$, and packet durations are exponentially distributed with mean $1 / \mu$.

A new optical packet arriving at the switch on wavelength $w$ and destined to output line $k$

- is forwarded to output line $k$ without using a TWC if channel $w$ is available, else

- is forwarded to output line $k$ using one of the free TWCs in the converter bank and using one of the free wavelength channels selected at random, else

- is blocked.

We concentrate only on one of the output fiber lines, say the $k$ th fiber, throughout this paper and study its packet blocking performance. The overall switch performance can then be obtained using the individual output fiber blocking probabilities since we use complete TWC partitioning across output fiber lines. We first assume that the aggregate optical packet arrival process from the $N$ input lines and destined for the output line $k$ is Poisson with rate $\lambda$. This assumption is very common especially when $N$ is large. For $W=K$ (i.e., full wavelength conversion), the OPS system behaves as an $M / M / c / c$ loss system with $c=K$ servers and offered load

$$
q=\lambda / \mu \text {. }
$$

On the other hand, the system load, or the traffic intensity is denoted by

$$
\rho=q / c
$$

in an $M / M / c / c$ system which describes the offered work load to a single server [30, page 13]. For $W=0$ (i.e, no wavelength conversion), we have $K$ independent single server $M / M / 1 / 1$ loss systems each with offered load $q=\lambda / \mu K$. In a general $M / M / c / c$ loss system, the blocking probability $P_{b}$ can be obtained using the Erlang B formula [30, page 80]:

$$
P_{b}=\frac{q^{c} / c !}{\sum_{i=0}^{c} q^{i} / i !} .
$$

A Markovian analysis for the partial wavelength conversion case, i.e., $0<W<K$, is described below. Let $i(t)$ and $j(t)$ denote the number of wavelength channels and the number of TWCs that are in use at time $t$, respectively. The process $X(t)=\{(i(t), j(t)): t \geq 0\}$ is a Markov process on the state space $S=\{(i, j): 0 \leq i \leq K, 0 \leq j \leq \min (i, W)\}$. To show this, let us assume that the process is in some state $(i, j), 0 \leq$ $i<K, 0 \leq j \leq \min (i, W)$ at time $t$. If a new packet arrives in the interval $(t, t+\delta t)$ which occurs with probability $\lambda \delta t+o(\delta t)$ (i.e., $\lim _{\delta t \rightarrow 0} o(\delta t) / \delta t=0$ ) [31, page 48], then there are three possibilities:

a1) the wavelength on which the burst is riding is not currently used on the link which occurs with probability $(K-i) / K$ and the burst will be admitted and the process will jump to $(i+1, j)$ at time $t+\delta t$,

a2) that wavelength is already used which occurs with probability $i / K$

a21) then if $j=W$, then the packet will be blocked because the converter pool is all busy leading to no state change, 
a22) else if $j<W$, then the packet will be admitted on one of the available wavelengths randomly using one of the free converters and the process will make a transition to state $(i+1, j+1)$ at time $t+\delta t$.

When the process is in state $(K, j)$ for some $j$ at time $t$, then the arriving burst will be blocked since all wavelengths are busy.

Assume now that the process $X(t)$ is currently in some state $(i, j), 0<i \leq K, 0 \leq j \leq \min (i, W)$ at time $t$. If a burst departs in the interval $(t, t+\delta t)$ which occurs with probability $i \mu \delta t+o(\delta t)$ then there are two possibilities:

b1) a TWC was used for this burst which occurs with probability $j / i$ and the process $X(t)$ will jump to state $(i-1, j-1)$ at at time $t+\delta t$,

b2) a TWC was not used for this departing burst which occurs with probability $(i-j) / i$ and the process $X(t)$ will make a transition to state $(i-1, j)$ at time $t+\delta t$.

When the process $X(t)$ is in state $(0,0)$, then there cannot be any departures. It is thus clear that the process $X(t)$ is a CTMC.

The next step is to write the infinitesimal generator of this CTMC in a form that is amenable to numerically stable and efficient computation of the steady-state probabilities. For this purpose, we decompose the state space into subsets called levels such that the number of wavelengths in use is constant within a level and we enumerate the states in the following order:

$$
\begin{aligned}
S=\{ & \{\underbrace{(0,0)}_{\text {level } 0}, \underbrace{(1,0),(1,1)}_{\text {level } 1}, \underbrace{(2,0),(2,1),(2,2)}_{\text {level } 2}, \cdots, \\
& \underbrace{(K, 0),(K, 1), \cdots,(K, W)}_{\text {level } K}\} .
\end{aligned}
$$

Based on this enumeration, we conclude that state transitions can occur either among neighboring levels or within a level. The final step is to express the infinitesimal generator of the process $X(t)$ based on the observations a1, a21, a22, b1, and b2. For this purpose, we define the following three matrices for $i \geq 0$ :

$$
\begin{aligned}
N_{i}= & {\left[\begin{array}{cccc}
\frac{K-i}{K} & \frac{i}{K} & & \\
& \frac{K-i}{K} & \frac{i}{K} & \\
& & \frac{K-i}{K} & \ddots \\
& & & \ddots
\end{array}\right], } \\
M_{i}= & {\left[\begin{array}{cccc}
i & & & \\
1 & i-1 & & \\
& 2 & i-2 & \\
& & \ddots & \ddots
\end{array}\right], } \\
\bar{I}_{i}= & {\left[\begin{array}{cccc}
0 & 0 & \ldots & 0 \\
0 & 0 & \ldots & 0 \\
\vdots & \vdots & \ddots & \vdots \\
0 & 0 & \ldots & i / K
\end{array}\right] . }
\end{aligned}
$$

We also let $I_{i}$ denote an identity matrix of size $i$ but we drop the subscript if the size is clear from the context. The process
$X(t)$ is then written as a CTMC with a block tridiagonal infinitesimal generator $Q$ which is in the following form:

$$
Q=\left[\begin{array}{ccccc}
A_{0} & U_{1} & & & \\
D_{0} & A_{1} & U_{2} & & \\
& D_{1} & A_{2} & \ddots & \\
& & \ddots & \ddots & U_{K} \\
& & & D_{K-1} & A_{K}
\end{array}\right] .
$$

In the above generator, it is not difficult to show by using a1, a21, and a22 that

$$
U_{i}=\lambda \bar{U}_{i}, \quad 0<i \leq K,
$$

where $\bar{U}_{i+1}$ equals

$\begin{cases}\text { upper-left }(W+1) \times(W+1) \text { block of } N_{i} & W \leq i, \\ \text { upper-left }(i+1) \times(i+2) \text { block of } N_{i} & 0 \leq i<W .\end{cases}$

On the other hand, $D_{i}$ in (5) is expressed as

$$
D_{i}=\mu \bar{D}_{i}, \quad 0 \leq i<K,
$$

where $\bar{D}_{i-1}$ equals

$\left\{\right.$ upper-left $(W+1) \times(W+1)$ block of $M_{i} \quad W<i$, upper-left $(i+1) \times i$ block of $M_{i} \quad 1 \leq i \leq W$.

Finally,

$$
A_{i}= \begin{cases}-(\lambda+i \mu) I_{i+1} & i<W, \\ -(\lambda+i \mu) I_{W+1}+\lambda \bar{I}_{i} & W \leq i<K, \\ -i \mu I_{W+1} & i=K .\end{cases}
$$

Steady-state probabilities of this irreducible and aperiodic CTMC can be found by solving for the unique stationary solution [31, pages 44-53]

$$
x Q=0, \quad x e=1,
$$

where $e$ is a column vector of ones of suitable size. Note that the size of $Q$ is $(W+1)\left(K-\frac{W}{2}+1\right)$ and calculating the stationary solution using conventional finite Markov chain solution methods (see for example the direct methods in [32, pages 61-117]) would be prohibitive especially for large systems, e.g., $K=256, W>>1$.

We now give a numerical solution procedure by taking advantage of the block tridiagonal structure of the generator. Since one of the equations in the first of the equations (9) is redundant, we can replace one such equation, say the first equation, to obtain a nonsingular system while preserving the block tridigonal structure. For this purpose, let $P$ be obtained by replacing the entries of the first column of $Q$ by setting

$$
A_{0}:=\lambda, D_{0}:=\left[\begin{array}{l}
0 \\
0
\end{array}\right],
$$

although we note that other choices are possible. Also let $b$ be a zero row vector except its first unity entry. It is clear that if $z$ is a solution to

$$
z P=b,
$$

then

$$
x:=\frac{z}{z e}
$$

gives the steady-state probabilities. 
We propose the block tridiagonal LU factorization algorithm given in [33, pages 174-175] for solving $z P=b$. In this algorithm, the goal is to obtain a block LU factorization of the matrix $P$ :

$$
P=\left[\begin{array}{cccc}
I & & & \\
L_{0} & I & & \\
& \ddots & \ddots & \\
& & L_{K-1} & I
\end{array}\right]\left[\begin{array}{cccc}
F_{0} & U_{1} & & \\
& F_{1} & \ddots & \\
& & \ddots & U_{K} \\
& & & F_{K}
\end{array}\right] .
$$

For this purpose, we partition the solution vectors $z=$ $\left(z_{0}, z_{1}, \ldots, z_{K}\right), x=\left(x_{0}, x_{1}, \ldots, x_{K}\right)$, and the right hand side of (11) $b=\left(b_{0}, b_{1}, \ldots, b_{K}\right)$ according to levels. Note that $b_{0}=1$ and $b_{i}=0,0<i \leq K$. The matrices $\left\{F_{i}\right\}, i=$ $0,1, \ldots, K$ and $\left\{L_{i}\right\}, i=0,1, \ldots, K-1$ can now be obtained using the following recurrence relation:

$$
\begin{aligned}
& F_{0}=A_{0} \\
& y_{0}=b_{0} F_{0}^{-1}\left(\text { Solve } y_{0} F_{0}=b_{0} \text { for } y_{0}\right) \\
& \text { for } i=1: K \\
& L_{i-1}=D_{i-1} F_{i-1}^{-1} \\
& F_{i}=A_{i}-L_{i-1} U_{i} \\
& y_{i}=\left(b_{i}-y_{i-1} U_{i}\right) F_{i}^{-1} \\
& \text { end }
\end{aligned}
$$

By backward substitution, one can then find $z_{k}, k=$ $0,1, \ldots, K$ :

$$
\begin{aligned}
& z_{K}=y_{K} \\
& \text { for } i=K-1: 0 \\
& \quad z_{i}=y_{i}-z_{i+1} L_{i} \\
& \text { end }
\end{aligned}
$$

Once $z_{k}$ s are computed, one can find the steady-state solution to (9) by using the identity (12). For numerical stability of the algorithm, we need a bound

$$
\max \left(\left\|L_{i}\right\|,\left\|F_{i}\right\|\right)<M, \forall i
$$

for some finite real number $M$ and for some legitimate matrix norm. The block LU factorization algorithm is known to be stable for block tridiagonal matrices that are block diagonally dominant by columns [34],[35]. We note that this condition is not met for the block tridiagonal matrix $P$. However, the matrix $P$ satisfies the block diagonal dominance by rows condition [34]:

$$
\left\|A_{i}^{-1}\right\|\left(\left\|U_{i+1}\right\|+\left\|D_{i-1}\right\|\right) \leq 1,0 \leq i \leq K,
$$

where $U_{K+1}$ and $D_{-1}$ are taken as zero matrices. Using the $\infty$ matrix norm from now on, we observe that the matrix $P$ is block diagonal dominant by rows since (14) is met for $i=0$ and $K$ and

$$
\left\|A_{i}^{-1}\right\|=\frac{1}{\lambda+i \mu},\left\|U_{i+1}\right\|=\lambda, 0<i<K
$$

and

$$
\left\|D_{i-1}\right\|=i \mu, 1<i<K,\left\|D_{0}\right\|=0 .
$$

When the block diagonal dominance by rows condition is met, it can be shown that [34]:

$$
\left\|F_{i}\right\| \leq\left\|A_{i}\right\|+\left\|U_{i+1}\right\|, 0 \leq i \leq K,
$$

and

$$
\left\|L_{i}\right\| \leq \frac{\left\|D_{i}\right\|}{\left\|U_{i+1}\right\|}, 0 \leq i \leq K-1 .
$$

Since $\left\|U_{i}\right\|=\lambda, 1 \leq i \leq K$ in our case, the proposed block tridiagonal LU factorization algorithm is numerically stable ensuring that the norms of the matrices $F_{i}$ and $L_{i}$ will not grow unreasonably large, i.e., these norms are in the order of the norms of the blocks of the original generator. Equivalently, this proposed method does not bring any additional instability when compared with conventional methods. Note that bulk of the computational effort is concentrated on the $\mathrm{LU}$ decomposition of the matrices $F_{i}$ s required in solving the linear systems in the block LU decomposition algorithm and the size of $F_{i}$ equals $i+1$ for $i \leq W$ and it is $W+1$ otherwise. Recalling that an LU decomposition requires $2 / 3 N^{3}$ flops for an $N \times N$ matrix, the proposed algorithm requires $2 / 3(W+1)^{2}\left(W^{2} / 4+(W+1)(K-W+1)\right)$ flops for all the LU decompositions which in turn gives rise to significant runtime and storage gains compared to the brute force approach.

We note that a new optical packet is blocked under the following two conditions upon the packet's arrival:

- the Markov chain resides in $(K, j), 0 \leq j \leq W$ (i.e. all wavelength channels are in use)

- the Markov chain resides in state $(i, W), W \leq i<K$ (i.e. all converters are in use) and the incoming wavelength is occupied (this occurs with probability $i / K$ )

The PASTA (Poisson Arrivals See Time Averages) property suggests that the steady-state probabilities at arbitrary times as calculated above are the same as those of the embedded discrete-time Markov chain at the epochs of arrivals [30, pages 221-222]. Therefore, the packet blocking probability $P_{b}$ can be expressed as

$$
P_{b}=x_{K} e+\sum_{i=W}^{K-1} \frac{i}{K} x_{i, W},
$$

where the solution vector for level $i$, namely $x_{i}$, is partitioned as $x_{i}=\left(x_{i, 0}, x_{i, 1}, \ldots, x_{i, W}\right)$ for $i \geq W$.

\section{ANAlysis OF SPL ARChitecture FOR THE Markovian ARrival Process CASE}

In this section, we aim at studying the impact of the packet arrival process characteristics on packet blocking performance. For doing so, we generalize our analytical model of Section II so as to cover also the MAP (Markovian Arrival Process) arrivals. The MAP generalizes the Poisson process by allowing non-exponential interarrival times but still maintaining its Markovian structure. The MAP is described by two processes, namely the the count process $N(t)$ and the phase process $J(t)$, assuming values in $\{0,1, \ldots\}$ and $\{0,1, \ldots, m-1\}$, respectively. The two-dimensional Markov process $\{N(t), J(t)\}$ is then modelled as a Markov process on the state-space 
$\{(i, j): i \geq 0,0 \leq j \leq m-1\}$ whose infinitesimal generator matrix $Q$ can be represented in block form as

$$
Q=\left[\begin{array}{ccccc}
C & D & & & \cdots \\
& C & D & & \cdots \\
& & C & D & \cdots \\
& & & & \ddots
\end{array}\right] .
$$

In the above generator, $C$ and $D$ are $m \times m$ matrices, $C$ has negative diagonal elements and non-negative off-diagonal elements, $D$ is non-negative, and $E=C+D$ is an irreducible infinitesimal generator. When the generator is of the form (16) then the underlying process is called MAP which is characterized by the matrix pair $(C, D)$. The evolution of the MAP is as follows. Assume that the Markov process with generator $E$ is in state $j, 0 \leq j \leq m-1$. After an exponentially distributed time interval with parameter $-C_{j j}$, there occurs either a transition to another state $k \neq j$ without an arrival with probability $\frac{C_{j k}}{-C_{j j}}$ or to a state $l$ (possibly the same state) with an arrival with probability $\frac{D_{j l}}{-C_{j j}}$. Let $\pi$ be the stationary probability vector of the phase process with generator $E$ so that $\pi$ satisfies

$$
\pi E=0, \pi e=1 .
$$

The mean arrival rate $\lambda$ for a MAP is given by [19]

$$
\lambda=\pi D e .
$$

Note that if we define

$$
d=D e=\left[\begin{array}{llll}
d_{0} & d_{1} & \cdots & d_{m-1}
\end{array}\right]^{T},
$$

then $d_{i}$ gives the arrival rate at phase $i$ and the mean arrival rate for the underlying MAP can then be written as $\lambda=$ $\sum_{i=0}^{m-1} \pi_{i} d_{i}$ which is equivalent to (18).

A subcase of MAP is PH-type which is widely used in the literature for modelling independent and identically distributed but non-exponential interarrival times [36, pages 44-46]. To describe a PH-type distribution, we define a Markov process on the states $\{0,1, \ldots, m\}$ with initial probability vector $(v, 0)$ and an infinitesimal generator

$$
\left[\begin{array}{cc}
T & T^{0} \\
0 & 0
\end{array}\right]
$$

where $v$ is a row vector of size $m$, the subgenerator $T$ is an $m \times m$ matrix, and $T^{0}$ is a column vector of size $m$ such that $T^{0}=-T e$. The distribution of the time till absorption into the absorbing state $m$ is called a $\mathrm{PH}$ distribution with representation $(v, T)$. If the interarrival times are modelled by $\mathrm{PH}$ distributions, then the arrival process is called a PH-type process. A PH-type process with representation $(v, T)$ is also a MAP by setting $C=T$ and $D=T^{0} v$.

Let us now study the optical packet switched link for the $0<W<K$ case first and with an arrival process modelled as a MAP with the characterizing pair of two $m \times m$ matrices $(C, D)$. It can be shown that the process $X(t)=\{(i(t), l(t), j(t)): t \geq 0\}$ on the state space $S=$ $\{(i, l, j): 0 \leq i \leq K, 0 \leq l \leq m-1,0 \leq j \leq \min (i, W)\}$, where $i(t)$ and $j(t)$ are defined as before and $l(t)$ is the phase of the MAP at time $t$, is also a CTMC. We enumerate the states as $S=$

$$
\begin{aligned}
& \{\underbrace{(0,0,0), \cdots,(0, m-1,0)}_{\text {level } 0}, \\
& \underbrace{(1,0,0),(1,0,1),(1,1,0), \cdots,(1, m-1,1)}_{\text {level } 1}, \cdots \\
& \underbrace{(K, 0,0), \cdots,(K, 0, W),(K, 1,0), \cdots,(K, m-1, W)}_{\text {level } K}\} .
\end{aligned}
$$

As before, we partition the solution vector $x=$ $\left(x_{0}, x_{1}, \ldots, x_{K}\right)$ according to levels. We also define the Kronecker product of an $m \times n$ matrix $A=\left\{a_{i j}\right\}$ and a $p \times q$ matrix $B$ to be an $m p \times n q$ matrix:

$$
A \otimes B=\left[\begin{array}{ccc}
a_{11} B & \cdots & a_{1 n} B \\
\vdots & \cdots & \vdots \\
a_{m 1} B & \cdots & a_{m n} B
\end{array}\right] .
$$

One can show that the resulting CTMC possesses an infinitesimal generator of the same block tridiagonal form (5) where

$$
\begin{gathered}
U_{i}=D \otimes \bar{U}_{i}, \\
D_{i}=\mu I_{m} \otimes \bar{D}_{i},
\end{gathered}
$$

and

$$
A_{i}= \begin{cases}\left(C-i \mu I_{m}\right) \otimes I_{i+1} & i<W, \\ \left(C-i \mu I_{m}\right) \otimes I_{W+1}+D \otimes \bar{I}_{i} & W \leq i<K, \\ \left(E-i \mu I_{m}\right) \otimes I_{W+1} & i=K .\end{cases}
$$

Therefore, the steady-state probabilities of this CTMC can be obtained using the same block LU factorization algorithm described in Section II. Finding these individual probabilities, one can find the packet blocking probability as follows. We first note that the PASTA property does not hold since the arrival process is not Poisson. However, when the system is at state $(i, l, W): W \leq i \leq K-1,0 \leq l \leq m-1$, then the converter pool is busy and an arriving packet will be blocked when it needs conversion which occurs with probability $i / K$. Partitioning $x_{i}, W \leq i \leq K$ as

$$
x_{i}=\left[x_{i, 0,0}, \ldots, x_{i, 0, W}, x_{i, 1,0}, \ldots, x_{i, m-1, W}\right],
$$

and recalling (19), the rate of blocked traffic at this state will be $i x_{i, l, W} d_{l} / K$. On the other hand, if the system is at state $(K, l, j): 0 \leq l \leq m-1,0 \leq j \leq W$, then the wavelength channels are all busy and an arriving packet will be dropped. The rate of blocked traffic in this state will then be $x_{K, l, j} d_{l}$. Summing up the rates of blocked traffic for all such states and dividing that to the mean arrival rate for the whole system, we find the probability that a single arriving packet will be lost, see also [36, page 94]. The above procedure can be formulated in terms of matrix operations for ease of computation. For this purpose, we first define the following two matrices

$$
G_{1}=D e \otimes\left[\begin{array}{c}
1 \\
\vdots \\
1 \\
1
\end{array}\right], G_{2}=I_{m} \otimes\left[\begin{array}{c}
0 \\
\vdots \\
0 \\
1
\end{array}\right] .
$$




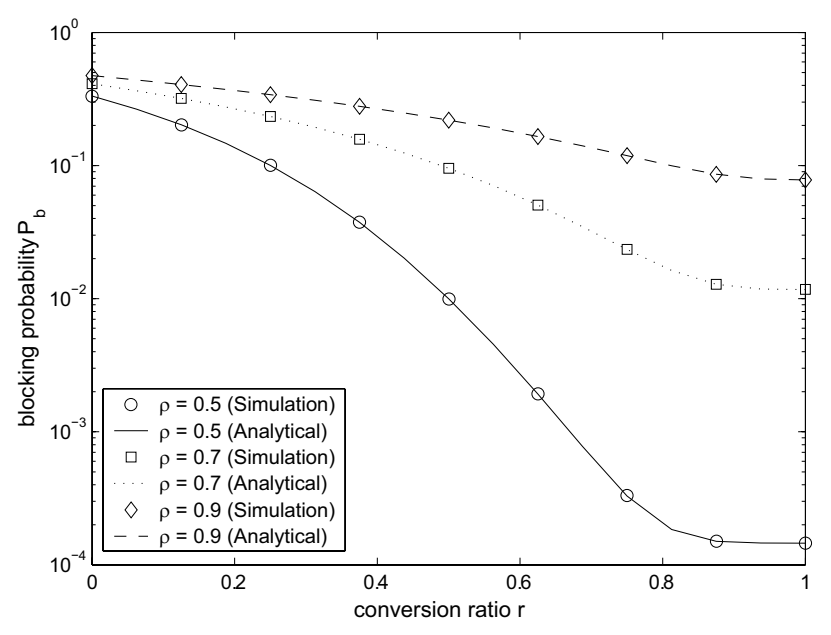

(a) $K=32$

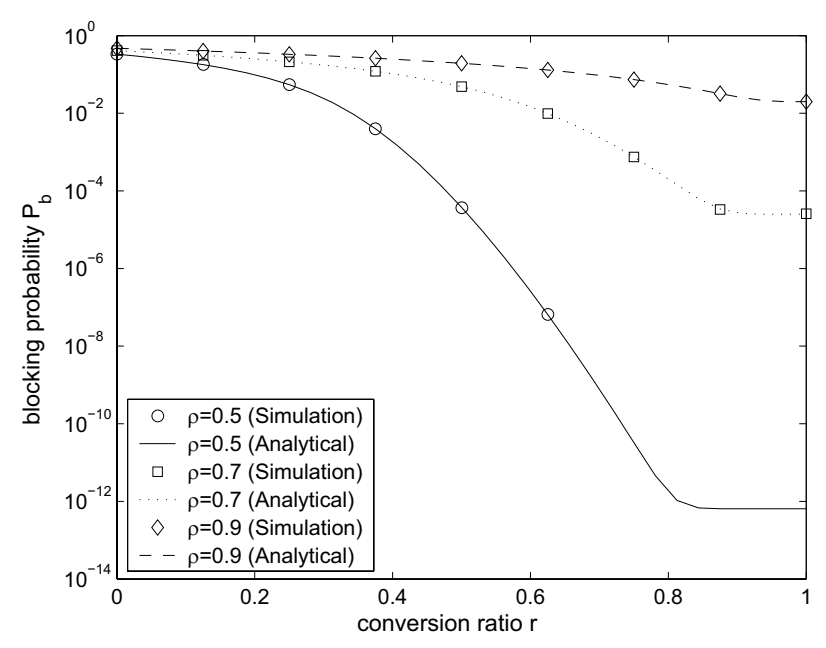

(b) $K=128$

Fig. 2. Blocking probability $P_{b}$ as a function of the wavelength conversion ratio $r$ for three different values of the system load $\rho$.

The packet blocking probability $P_{b}$ can then be written as

$$
P_{b}=\frac{x_{K} G_{1}+\sum_{i=W}^{K-1} \frac{i}{K} x_{i} G_{2} D e}{\pi D e} .
$$

The above analysis applies to $0<W<K$. The special cases of $W=0$ and $W=K$ are studied next.

\section{A. No Wavelength Conversion}

In case of NWC (i.e., $W=0$ ), we have $K$ independent servers each fed with a MAP that is obtained by splitting the aggregate MAP stream $(C, D)$ evenly into $K$ substreams. The characterizing pair of matrices $\left(C_{N W C}, D_{N W C}\right)$ for each substream is given by

$$
C_{N W C}=C+\frac{K-1}{K} D, D_{N W C}=\frac{1}{K} D .
$$

The infinitesimal generator for the underlying $M A P / M / 1 / 1$ system for each wavelength channel is then written as

$$
Q=\left[\begin{array}{cc}
C_{N W C} & D_{N W C} \\
\mu I_{m} & E-\mu I_{m}
\end{array}\right]
$$

Solving the steady state probabilities for (26) through

$$
x Q=0, x e=1, x=\left(x_{0}, x_{1}\right),
$$

the blocking probability for each channel can be written as

$$
P_{b}=\frac{x_{1} D_{N W C} e}{\pi D_{N W C} e} .
$$

\section{B. Full Wavelength Conversion}

The other boundary at $W=K$ is the full wavelength conversion case and the loss system then gives rise to the so-called $M A P / M / K / K$ system. This process has the infinitesimal generator of the form (5) where

$$
\begin{aligned}
D_{i} & =(i+1) \mu I_{m}, 0 \leq i<K, \\
A_{i} & =C-i \mu I_{m}, 0 \leq i<K, \quad A_{K}=E-K \mu I_{m}, \\
U_{i} & =D, 1 \leq i \leq K .
\end{aligned}
$$

Solving for the steady-state probabilities of this CTMC and partitioning the solution as $x=\left(x_{0}, x_{1}, \ldots, x_{K}\right)$, the blocking probability for the $M A P / M / K / K$ system is given by

$$
P_{b}=\frac{x_{K} D e}{\pi D e} .
$$

\section{NUMERICAL RESULTS}

The exact analysis method introduced in this paper for calculating the blocking probabilities in packet switching nodes is implemented using Matlab. Without loss of generality, we set the mean packet length $1 / \mu$ to unity in all the numerical examples. We use a $\mathrm{C}++$ based event driven program to carry out the simulations. Each simulation result for the blocking probability is obtained via the mean of ten independent runs where an overall of $10^{9}$ optical packets are simulated at each run. We keep track of the occupancy of each individual wavelength in the simulations. To generate MAPs, we use the description of the MAP at the beginning of Section III.

We first assume that the packet arrival process is Poisson. Using the expression (15), the packet blocking probabilities are analytically calculated for the two cases $K=32$ and $K=128$ as a function of the wavelength conversion ratio

$$
r:=W / K
$$

and for three different system loads $\rho=0.5,0.7$, and 0.9 , and the results are compared against simulations. We present our results in Fig. 2. For all the tested cases, the analytical results are in perfect accordance with the simulation results, validating the proposed approach for Poisson traffic. We note that simulations are not performed in cases where rare probabilities such as $P_{b}<10^{-8}$ need to be simulated due to excessive simulation run-times that would be needed.

In the second example, we plot the blocking probability $P_{b}$ as a function of $r$ and $\rho$ in Fig. 3 in the form of a 3-dimensional mesh for three different cases of $K=64,128$, and 256, respectively. This plot shows that we can obtain blocking probabilities for very large systems (e.g., $K=256$ ) and for very rare probabilities (e.g., $P_{b} \approx 10^{-40}$ ) and for a wide 


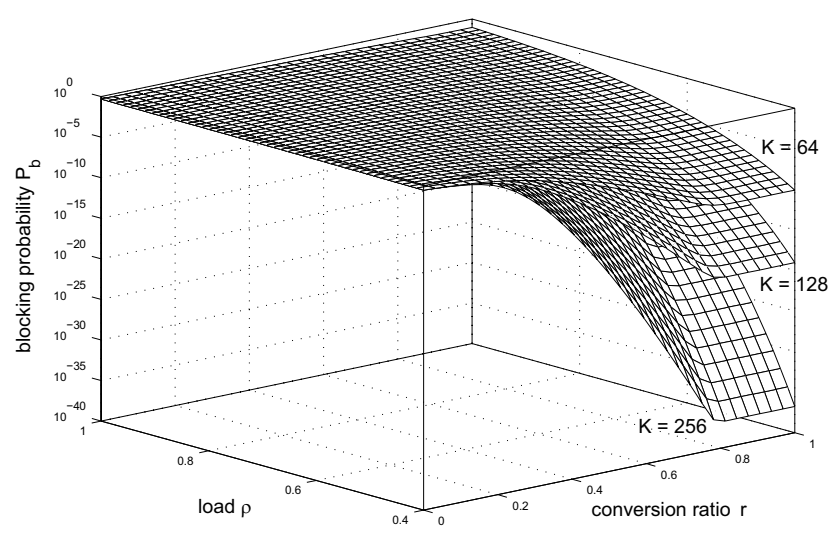

Fig. 3. Blocking probability $P_{b}$ as a function of the wavelength conversion ratio $r$ and system load $\rho$ for three different values of $K$.

range of system parameters (i.e., $0.4<\rho<1,0<r<1$ ) without encountering any numerical problems. Moreover, such probabilities can be obtained rather rapidly. Plotting the three planes on Fig. 3 required solution of 4554 problems described in Section II and obtaining Fig. 3 consisting of three planes took less than $11 / 2$ hours on a $3 \mathrm{GHz}$ Pentium based PC. We believe that rapid generation of these plots can be very helpful for converter provisioning purposes as will be described later in the sequel.

The computation times for the case $K=128$ and $\rho=0.5$ are plotted in Fig. 4(a) as a function of the number of converters $W$. We observe a faster than linear growth in the execution times when $W$ is small followed by an almost linear behavior in execution times for a wide range of $W$. The execution times tend to grow slower than linear when $W$ approaches $K$. The conclusion we draw from this observation is that growth in the size of the converter bank does not pose computational problems as they would in algorithms with polynomial complexity. For the dual case with $W=80$ and $q=64$, we observe that the computation times tend to be linear in $K$ as depicted in Fig. 4(b).

\section{A. Effect of Packet Interarrival Time Distribution}

We assume that the packet arrival process is phase type and we study the impact of the Squared Coefficient of Variation (SCV), denoted by $\gamma$, of packet interarrival times on packet blocking performance [37]. The SCV of a random variable is the variance divided by the squared mean of that random variable and is indicative of its variability. For Poisson arrivals $\gamma=1$, whereas for $\gamma<1$ we use the Erlang- $k$ distribution which has $\gamma=1 / k$ and which has $k$ phases. On the other hand, the cases of $\gamma>1$ can be obtained by using an appropriate two phase hyperexponential distribution (denoted by $\mathrm{H}_{2}$ ) with balanced means [37, pages 58-59]. Fig. 5 depicts the packet blocking probabilities as a function of the wavelength conversion ratio for $K=64$, under two different loads and for different values of the SCV of the arrival process. Again for the PH-type case, we obtain a perfect match of the analytical results with those obtained using simulations. We conclude that packet blocking probabilities are significantly lower for regularly spaced arrivals with relatively small SCV. This observation demonstrates that not only the mean but also the variance of packet interarrival times have a substantial

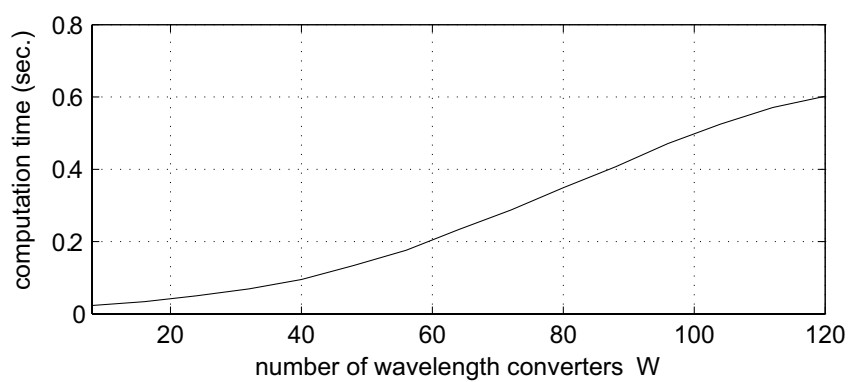

(a)

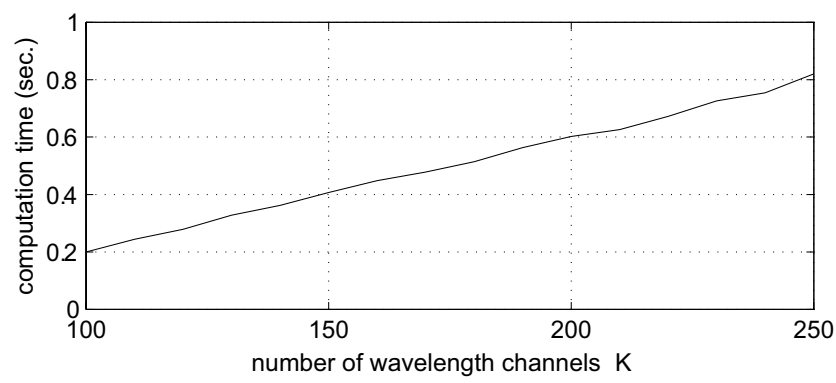

(b)

Fig. 4. Computation times for (a) with respect to varying $W$ for $K=128$ (b) with respect to varying $K$ when $W=80$.

impact on packet blocking performance and these second order traffic characteristics need to be taken into consideration for accurately modelling optical packet switching systems. We also conclude that traffic shaping at the ingress of an OPS network that can reduce the SCV would also be effective in reducing packet blocking inside the OPS core.

\section{B. Effect of Packet Length Distribution}

It is also interesting to study whether the blocking probabilities are sensitive to the packet length distribution for the Poisson arrival case. Recall that in the Erlang loss systems, the service time distribution influences the blocking probability only through its mean, and the higher-order statistics do not affect the blocking probabilities. Since the boundary cases of $W=0$ and $W=K$ reduce to Erlang loss systems of some form, one might lead to the conclusion that the blocking probabilities in the general PWC case, i.e., $0<$ $W<K$, is also insensitive to the higher order statistics of the packet length distribution. To study this phenomenon, we take three packet length distributions (exponential, deterministic, hyperexponential) all with the same mean but with different SCV values. We then fix $K=32$, and for different values of $W$ and $\rho$ we obtain $P_{b}$ but we resort to simulations for non-exponential packet length distributions. The results are presented in Table I. We observe that the blocking probabilities for the PWC system with deterministic and hyperexponential packet lengths deviate slightly from the one with exponential packet lengths especially under the regime of low load and moderate number of TWCs. These results lead us to believe that the corresponding insensitivity may not be perfect for PWC as in the Erlang loss systems but one can still make use of exponential packet length distributions for approximating the behavior of non-exponential burst lengths. However, we note that studying the sensitivity to packet length distributions for more general MAP arrivals is left for future research. 


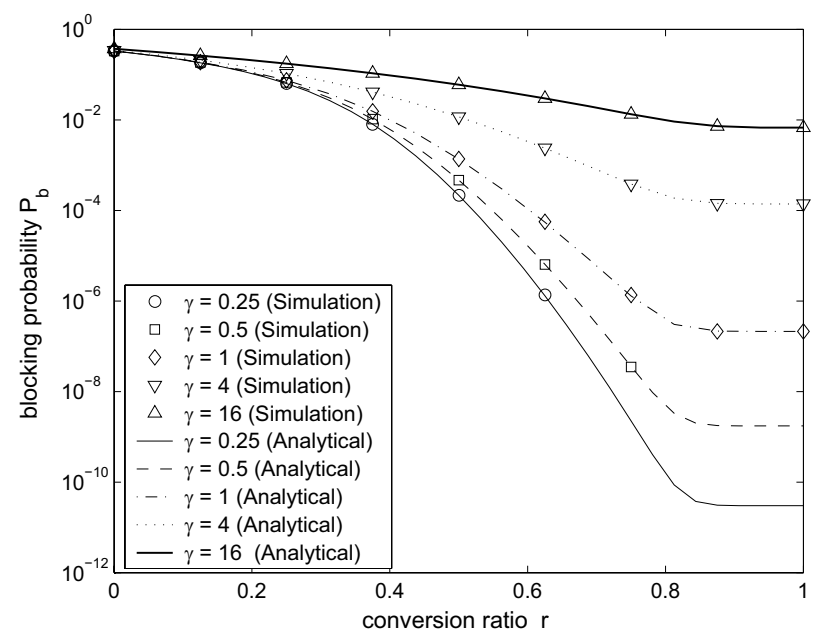

(a) $\rho=0.5$

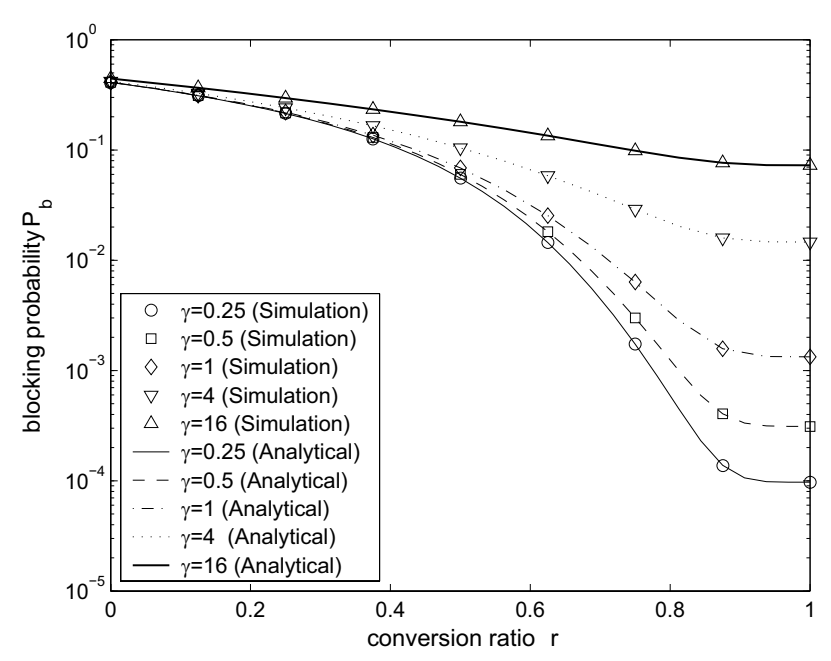

(b) $\rho=0.7$

Fig. 5. Blocking probability $P_{b}$ for $K=64$ as a function of $r$ for different values of the squared coefficient of variation $\gamma$.

TABLE I

PACKET BLOCKING PROBABILITIES FOR EXPONENTIAL, DETERMINISTIC AND HYPEREXPONENTIAL PACKET LENGTH DISTRIBUTIONS IN A NUMBER OF SCENARIOS

\begin{tabular}{|c|c|c|c|}
\hline \multirow[t]{2}{*}{$W$} & $\mathrm{SCV}=1$ & $\begin{array}{c}\mathrm{SCV}=0 \\
(\% 95 \text { Conf. Intervals })\end{array}$ & $\begin{array}{c}\text { SCV }=4 \\
\text { (\%95 Conf. Intervals) }\end{array}$ \\
\hline & \multicolumn{3}{|c|}{$\rho=0.4$} \\
\hline 4 & $1.32 \mathrm{E}-01$ & 1.33E-01 $( \pm 3.38 \mathrm{E}-05)$ & $1.31 \mathrm{E}-01( \pm 6.82 \mathrm{E}-05)$ \\
\hline 12 & $7.37 \mathrm{E}-03$ & $8.14 \mathrm{E}-03( \pm 1.39 \mathrm{E}-05)$ & $7.01 \mathrm{E}-03( \pm 2.27 \mathrm{E}-05)$ \\
\hline 20 & $6.76 \mathrm{E}-05$ & 8.97E-05 ( \pm 1.39E-06 $)$ & $5.59 \mathrm{E}-05( \pm 1.22 \mathrm{E}-06)$ \\
\hline \multirow[t]{2}{*}{28} & $2.86 \mathrm{E}-06$ & $2.95 \mathrm{E}-06( \pm 2.89 \mathrm{E}-07)$ & $2.82 \mathrm{E}-06( \pm 1.27 \mathrm{E}-07)$ \\
\hline & \multicolumn{3}{|c|}{$\rho=0.6$} \\
\hline 4 & $2.65 \mathrm{E}-01$ & $2.66 \mathrm{E}-01( \pm 7.13 \mathrm{E}-05)$ & $2.65 \mathrm{E}-01( \pm 1.05 \mathrm{E}-04)$ \\
\hline 12 & $9.25 \mathrm{E}-02$ & 9.47E-02 $( \pm 8.57 \mathrm{E}-05)$ & $9.18 \mathrm{E}-02( \pm 7.61 \mathrm{E}-05)$ \\
\hline 20 & $1.49 \mathrm{E}-02$ & $1.65 \mathrm{E}-02( \pm 5.18 \mathrm{E}-05)$ & $1.43 \mathrm{E}-02( \pm 3.68 \mathrm{E}-05)$ \\
\hline 28 & $2.17 \mathrm{E}-03$ & $2.24 \mathrm{E}-03( \pm 1.48 \mathrm{E}-05)$ & $2.12 \mathrm{E}-03( \pm 9.25 \mathrm{E}-06)$ \\
\hline
\end{tabular}

\section{Effect of Packet Arrival Process Correlation}

Another issue we study is the impact of the arrival process correlation on packet blocking probabilities. For this purpose, we use the method of [38] which introduces a correlation into an arbitrary uncorrelated arrival process without changing the marginals. As an example if we have a PH-type distribution characterized by the pair $(v, T)$ with $T^{0}=-T e$, then the MAP defined by

$$
C=T, D=(1-\psi) T^{0} v-\psi T, 0 \leq \psi<1
$$

has a lag-k autocorrelation between the $i$ th and $i+k$ th interarrival times in the form $c \psi^{k}$ for some constant $c$. A large value of the correlation parameter $\psi$ implies a slow decay of the lag-k autocorrelation function and therefore strong correlation. On the other hand, a small $\psi$ yields weakened autocorrelation and in the limit when $\psi=0$ we obtain back the uncorrelated phase-type process. As an example, we take an arrival process with hyperexponential (with balanced means) marginals with $\gamma=16$ and we incorporate autocorrelation into this process as described above. In Fig. 6, the packet blocking probability is plotted with respect to the wavelength conversion ratio $r$ for three different correlation parameter values $\psi$ in a PWC system with $K=64$ and for two different cases stemming from two different choices of $\rho$. The results are again in accordance with the simulation results in all cases validating our proposed approach for MAP arrivals. Moreover, increase in the correlation parameter $\psi$ also substantially increases the packet blocking probability as would be expected whereas the impact is more severe with increasing wavelength conversion ratio.

\section{Tradeoff Between Number of Channels and Converters}

We utilize this fast and exact blocking probability analysis technique for finding the minimum cost combination of number of wavelength channels and converters for achieving a given Grade of Service (GoS) requirement in optical packet switching systems. While doing so, we assume arrivals are Poisson in all the examples although the proposed procedure can be applied to more general MAP arrivals as well. For Fig. 7-9, we assume a fixed $\lambda=15$. For a varying number of wavelengths per fiber (i.e., $K$ ), we iteratively find the minimum number of wavelength converters that meets a GoS 


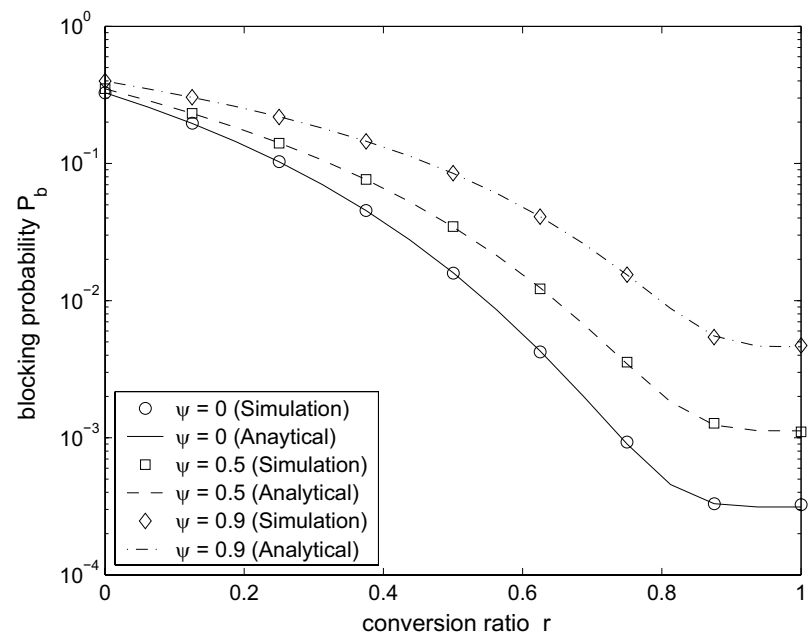

(a) $\rho=0.4$

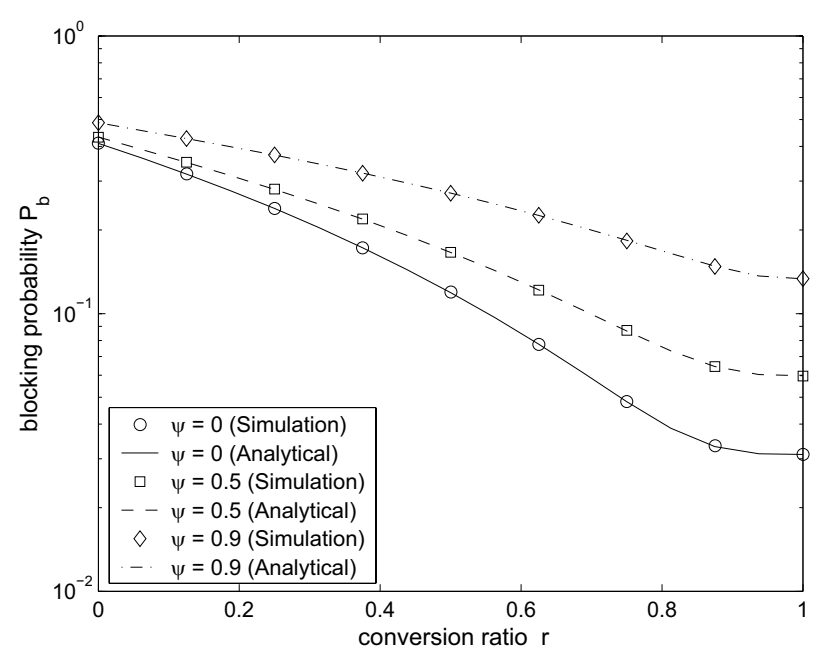

(b) $\rho=0.6$

Fig. 6. Blocking probability $P_{b}$ for $K=64$ as a function of the wavelength conversion ratio $r$ for different values of the system load $\rho$ and correlation parameter $\psi$.

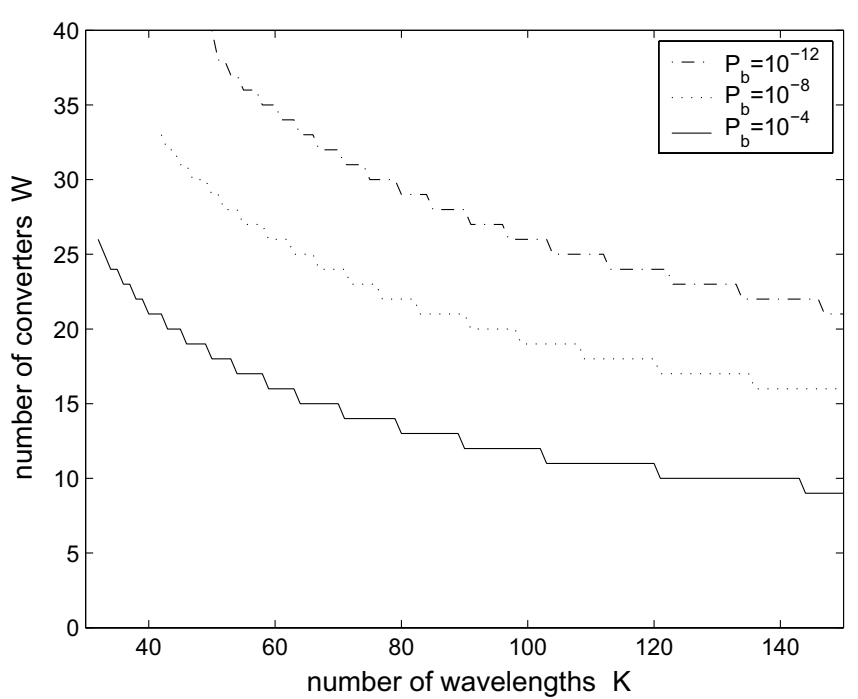

Fig. 7. Number of converters required for a given $P_{b}$ as a function of the number of wavelength channels $K$.

requirement expressed in terms of $P_{b}$. The results are depicted for different values of $P_{b}$ in Fig. 7. The region above each curve in Fig. 7 represents the feasibility set composed of $(K, W)$ pairs satisfying the GoS requirement. For a fixed offered traffic and target GoS expressed in terms of $P_{b}$, the trade-off between the number of wavelengths and number of converters is illustrated in Fig. 7. From Fig. 7, we observe that lack of converters can be compensated by increasing the number of wavelength channels in order to achieve a target GoS.

This trade-off is further investigated in Fig. 8 from the switch cost point of view. We assume that a TWC has a cost which is $\alpha$ times larger than the wavelength channel cost. The points indicated in Fig. 8 are the optimum combination of $(K, W)$ resulting in the minimum switch cost with $P_{b}=10^{-4}$ for three different values of $\alpha, \alpha=2,6,15$. Each optimum point corresponds to the intersection of the equi-cost line of

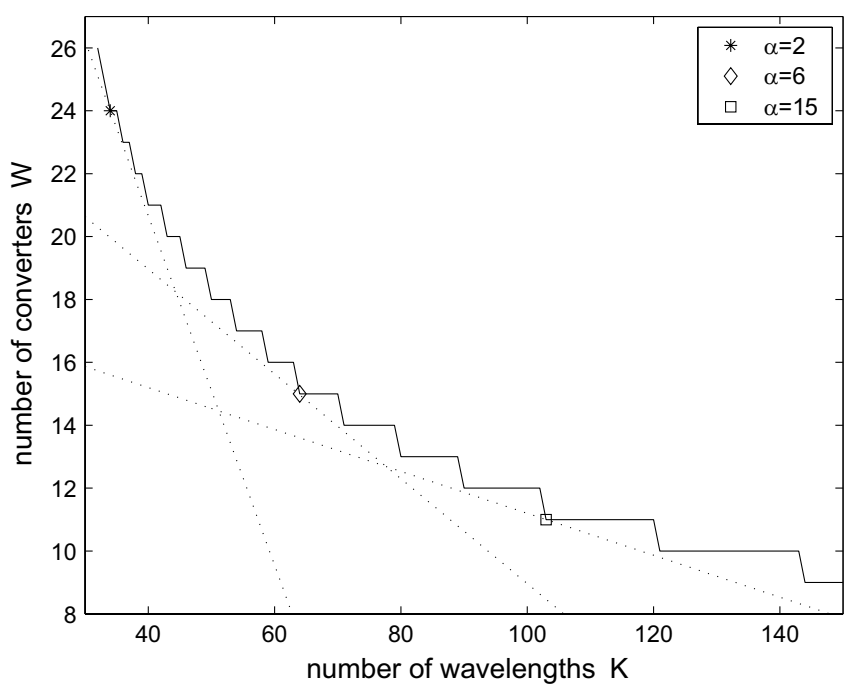

Fig. 8. Optimum operating point $(K, W)$ for $P_{b}=10^{-4}$ as a function the cost parameter $\alpha$.

slope $-1 / \alpha$ that is tangent to the boundary of the feasibility region for $P_{b}=10^{-4}$. As the cost of TWC increases, the optimum operation point moves to a regime where fewer number of converters are used in conjunction with a larger number of wavelength channels. The least cost solution has a conversion ratio $r$ of about two thirds when TWCs are relatively inexpensive $(\alpha=2)$. On the other hand, with relatively high cost of conversion $(\alpha=15)$, conversion ratio $r$ reduces to about $10 \%$ in the optimum solution.

The effects of $\alpha$ and $P_{b}$ on the optimum conversion ratio are studied in Fig. 9. We observe that the optimum conversion ratio quickly drops as $\alpha$ increases for different GoS constraints, but the decrease rate slows down as $\alpha$ becomes prohibitively high. The optimum conversion ratio increases as the GoS constraint becomes more stringent, however the dependence is not very strong, i.e., the conversion ratio is relatively insensitive with respect to the target $P_{b}$. 


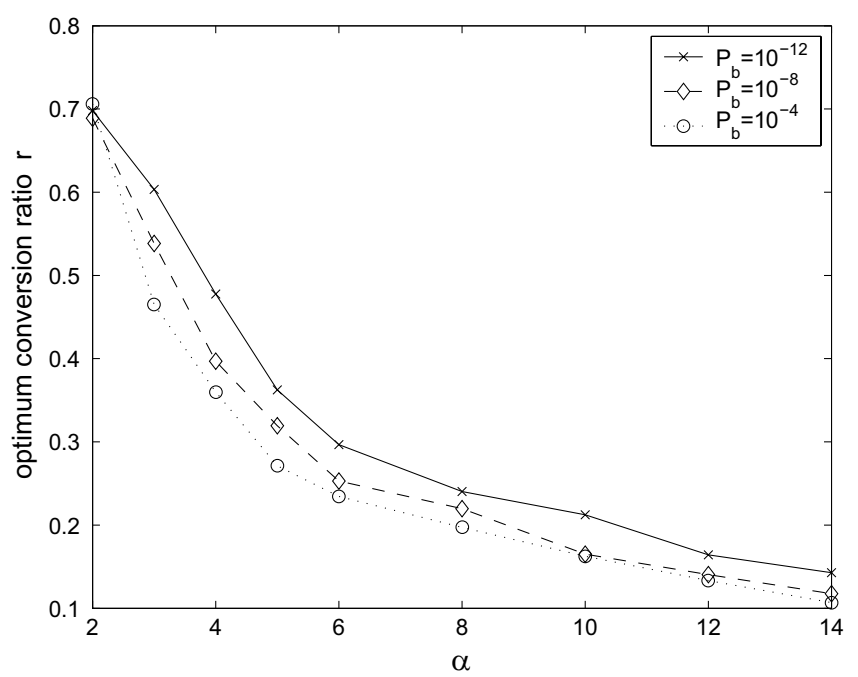

Fig. 9. Optimum conversion ratio as a function of the cost parameter $\alpha$ for $P_{b}=10^{-4}, 10^{-8}$ and $10^{-12}$.

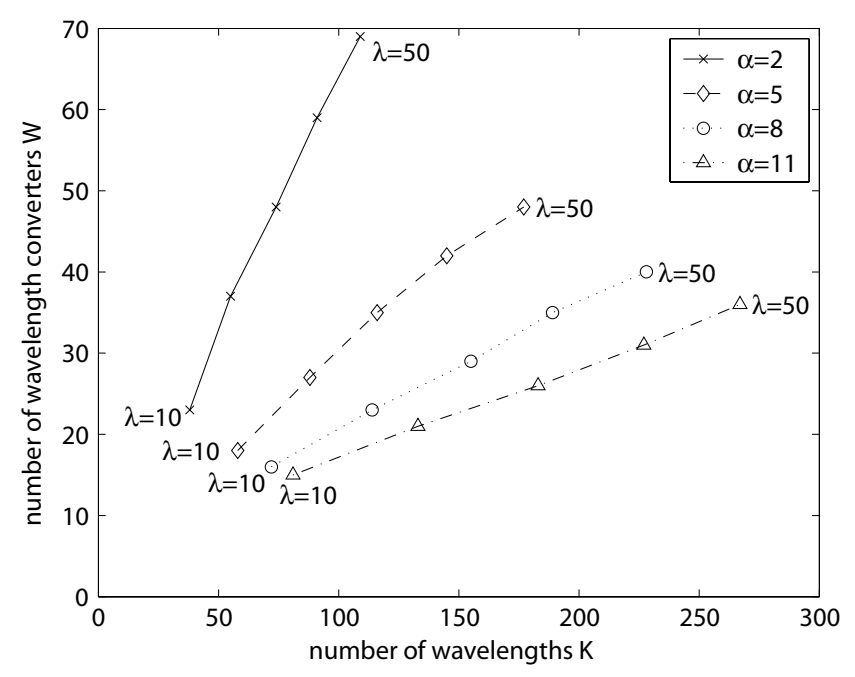

Fig. 10. Optimum operating points $(K, W)$ for $P_{b}=10^{-8}$ as a function the arrival rate $\lambda$ for different values of cost parameter $\alpha$.

Finally, the effect of the Poisson arrival rate $\lambda$ on the optimum combination of $(K, W)$ for different values of $\alpha$ is depicted in Fig. 10 for $P_{b}=10^{-8}$. In this figure, $\lambda$ is increased from 10 to 50 in steps of 10 . It is observed that as $\lambda$ increases, the optimum $(K, W)$ pair moves approximately along a line passing through the origin for all values of $\alpha$ we tested. The rate of this increase decreases with increasing $\alpha$ since the minimum cost switch uses fewer converters as $\alpha$ increases. Consequently, we conclude that increasing $\lambda$ has a minor effect on the optimum conversion ratio when $\alpha$ remains constant.

\section{CONCLUSION}

We propose an exact analytical method for exactly calculating blocking probabilities in OBS/OPS nodes employing shared wavelength conversion on a share-per-link basis. The method is first formulated for Poisson arrivals and then extended to the more general Markovian arrival process which is commonly used for traffic modelling in the Internet. The proposed exact analysis method can be used for efficiently and accurately calculating blocking probabilities even in very large systems with hundreds of wavelength channels and rare blocking probabilities. Moreover, using the analysis for Markovian arrivals, we show that the variability of the interarrival times and the correlation in the packet traffic substantially impact the blocking probabilities in asynchronous optical packet switching systems and such second-order statistics also need to be taken into account. The rapid calculation of the packet blocking probabilities allows optimum provisioning of bufferless OPS links when the cost relationship between a wavelength channel and a wavelength converter is available. Using an optimum provisioning procedure, we also study the effects of arrival rate, GoS constraint and the converter cost on the optimum sizing of optical packet switching systems.

\section{REFERENCES}

[1] P. Gambini, M. Renaud, C. Guillemot, F. Callegati, I. Andonovic, B. Bostica, D. Chiaroni, G. Corazza, S. L. Danielsen, P. Gravey, P. B. Hansen, M. Henry, C. Janz, A. Kloch, R. Krahenbuhl, C. Raffaelli, M. Schilling, A. Talneau, and L. Zucchelli, "Transparent optical packet switching: network architecture and demonstrators in the KEOPS project," IEEE J. Sel. Areas Commun., vol. 16, no. 7, pp. 1245-1259, 1998.

[2] G. N. Rouskas and L. Xu, "Optical packet switching," in Emerging Optical Network Technologies: Architectures, Protocols, and Performance, K. Sivalingam and S. Subramaniam, Eds. Norwell, MA: Springer, 2004, pp. 111-127.

[3] C. Qiao and M. Yoo, "Optical burst switching (OBS): a new paradigm for an optical Internet," J. High Speed Networks (JHSN), vol. 8, no. 1, pp. 69-84, 1999.

[4] Y. Chen, C. Qiao, and X. Yu, "Optical burst switching: a new area in optical networking research," IEEE Network Mag., vol. 18, no. 3, pp. $16-23,2004$.

[5] R. Ramaswami and K. N. Sivarajan, Optical Networks: A Practical Perspective, Second Edition. Morgan Kaufmann Publishers, 2002.

[6] S. Yao, S. J. B. Yoo, B. Mukherjee, and S. Dixit, "All-optical packet switching for metropolitan area networks: opportunities and challenges," IEEE Commun. Mag., vol. 39, no. 3, pp. 142-148, 2001.

[7] V. Eramo, M. Listanti, and P. Pacifici, "A comparison study on the wavelength converters number needed in synchronous and asynchronous all-optical switching architectures," IEEE J. Lightwave Technol., vol. 21, no. 2, pp. 340-355, 2003.

[8] R. A. Barry and P. A. Humblet, "Models of blocking probability in alloptical networks with and without wavelength changers," IEEE J. Sel. Areas Commun., vol. 14, no. 5, pp. 858-867, 1996.

[9] E. Karasan and E. Ayanoglu, "Effects of wavelength routing and selection algorithms on wavelength conversion gain in WDM optical networks," IEEE/ACM Trans. Networking, vol. 6, no. 2, pp. 186-196, 1998.

[10] M. Kovacevic and A. Acampora, "Benefits of wavelength translation in all-optical clear channel networks," IEEE J. Sel. Areas Commun., vol. 14, no. 5, pp. 868-880, 1996.

[11] K. Lee and V. Li, "A wavelength convertible optical network," IEEE J. Lightwave Technol., vol. 11, no. 5, pp. 962-970, 1993.

[12] V. Eramo and M. Listanti, "Input wavelength conversion in optical packet switches," IEEE Commun. Lett., vol. 7, no. 6, pp. 281-283, 2003.

[13] D. Mitra, C. Nuzman, I. Saniee, and P. Whiting, "Optical cross-connect with shared wavelength conversion under dynamic load," IEEE/OFC Technical Digest, pp. 160-162, 2002.

[14] N. Akar and E. Karasan, "Exact calculation of blocking probabilities for bufferless optical burst switched links with partial wavelength conversion," in Proc. 1st Conference on Broadband Networks (BROADNETS'04), Optical Networking Symposium, pp. 110-117.

[15] Y. Mingwu, L. Zengji, and W. Aijun, "Accurate and approximate evaluations of asynchronous tunable-wavelength-converter sharing schemes in optical burst-switched networks," IEEE J. Lightwave Technol., vol. 23, no. 10 , pp. $2807-2815,2005$.

[16] C. M. Gauger, "Performance of converter pools for contention resolution in optical burst switching," in Proc. SPIE Optical Networking and Communications Conference (OptiComm 2002). 
[17] J. Spath and S. Bodamer, "Routing of dynamic Poisson and non-Poisson traffic in WDM networks with limited wavelength conversion," in Proc. 24th European Conference on Optical Communication, 1998.

[18] D. M. Lucantoni, K. S. Meier-Hellstern, and M. F. Neuts, "A single server queue with server vacations and a class of nonrenewal arrival processes," Adv. Applied Prob., vol. 22, no. 3, pp. 676-705, 1990.

[19] D. M. Lucantoni, "New results for the single server queue with a batch Markovian arrival process," Stochastic Models, vol. 7, no. 1, pp. 1-46, 1991.

[20] M. F. Neuts, Structured Stochastic Matrices of M/G/1 Type and Their Applications. New York: Marcel Dekker, 1989.

[21] G. Latouche and V. Ramaswami, Introduction to Matrix Analytical Methods in Stochastic Modeling. ASA-SIAM Series on Statistics and Applied Probability, 2002.

[22] A. Kuczura, "The interrupted Poisson process as an overflow process," Bell Sys. Tech. J., vol. 52, no. 3, pp. 437-448, 1973.

[23] A. Riska, V. Diev, and E. Smirni, "Efficient fitting of long-tailed data sets into phase-type distributions," SIGMETRICS Perform. Eval. Rev., vol. 30, no. 3, pp. 6-8, 2002.

[24] H. Heffes and D. Lucantoni, "A Markov modulated characterization of packetized voice and date traffic and related statistical multiplexer performance," IEEE J. Sel. Areas Commun., vol. 4, no. 6, pp. 856-868, 1986.

[25] D. P. Heyman and D. Lucantoni, "Modeling multiple IP traffic streams with rate limits," IEEE/ACM Trans. Networking, vol. 11, no. 6, pp. 948958, 2003.

[26] P. Salvador, R.Valadas, and A. Pacheco, "Multiscale fitting procedure using Markov modulated Poisson processes," Telecommunication Systems, vol. 23, no. 1-2, pp. 123-148, 2003.

[27] L. Muscariello, M. Mellia, M. Meo, R. Lo Cigno, and M. A. Marsan, "An MMPP-based hierarchical model of Internet traffic," in Proc. ICC 2004.

[28] G. M. Lee, J. K. Choi, B. P. Wydrowski, M. Zukerman, and C.-H. Kang, "Optical hybrid switching: combined optical burst switching and optical circuit switching," in Proc. ICOIN 2004, pp. 740-749.

[29] H. Overby, "How the packet length distribution influences the packet loss rate in an optical packet switch," in Proc. Advanced International Conference on Telecommunications and International Conference on Internet and Web Applications and Services (AICT/ICIW 2006), p. 46.

[30] D. Gross and C. M. Harris, Fundamentals of Queueing Theory, Third Edition. New York: Wiley, 1997.

[31] L. Kleinrock, Queuing Systems, Vol. 1, Theory. New York: John Wiley, 1989.

[32] W. J. Stewart, Introduction to the Numerical Solution of Markov Chains. Princeton University Press, 1994.

[33] G. H. Golub and C. F. van Loan, Matrix Computations, Third Edition. The Johns Hopkins University Press, 1996.

[34] J. M. Varah, "On the solution of block-tridiagonal systems arising from certain finite difference equations," Mathematics of Computation, vol. 26, no. 120 , pp. 859-868, 1972.

[35] J. W. Demmel, N. J. Higham, and R. S. Schreiber, "Stability of block LU factorization," Numerical Lin. Alg. and Appl., vol. 2, no. 2, pp. 173-190, 1995.

[36] M. F. Neuts, Matrix-Geometric Solutions in Stochastic Models. Baltimore, MD: Johns Hopkins University Press, 1989.

[37] B. R. Haverkort, Performance of Computer and Communication Systems: A Model-based Approach. New York: John Wiley and Sons, 1998.
[38] K. Mitchell, "Constructing a correlated sequence of matrix exponentials with invariant first-order properties," Operations Research Lett., vol. 28, no. 1, pp. 27-34, 2001.

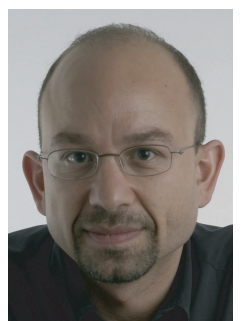

Nail Akar received his B.S. degree from Middle East Technical University, Turkey, in 1987 and M.S and Ph.D. degrees from Bilkent University, Turkey, in 1989 and 1994, respectively, all in electrical and electronics engineering.

From 1994 to 1996 , he was a visiting scholar and a visiting assistant professor in the Computer Science Telecommunications program at the University of Missouri-Kansas City. In 1996, he joined the Technology Planning and Integration group at the Long Distance Division, Sprint, where he held a senior member of technical staff position from 1999 to 2000. Since 2000, he has been a faculty member at Bilkent University, currently as an associate professor. His current research interests include performance analysis of computer and communication networks, queuing systems, traffic engineering, network control and resource allocation, and optical networking.

Dr. Akar is on the current editorial board of Computer Networks and he actively participates in European Commission FP6 NoE projects ePhoton/ONe+ and NEWCOM.

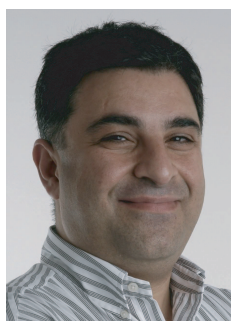

Ezhan Karasan received B.S. degree from Middle East Technical University, Ankara, Turkey, M.S. degree from Bilkent University, Ankara, Turkey, and Ph.D. degree from Rutgers University, Piscataway, New Jersey, USA, all in electrical engineering, in 1987, 1990, and 1995, respectively. During 19951996, he was a post-doctorate researcher at Bell Labs, Holmdel, New Jersey, USA. From 1996 to 1998, he was a Senior Technical Staff Member in the Lightwave Networks Research Department at AT\&T Labs-Research, Red Bank, New Jersey, USA. He has been with the Department of Electrical and Electronics Engineering at Bilkent University since 1998, where he is currently an associate professor. During 1995-1998, he worked in the Long Distance Architecture task of the Multiwavelength Optical Networking Project (MONET), sponsored by DARPA. Dr. Karasan is a member of the Editorial Board of Optical Switching and Networking journal. He is the recipient of 2004 Young Scientist Award from Turkish Scientific and Technical Research Council (TUBITAK), 2005 Young Scientist Award from Mustafa Parlar Foundation and Career Grant from TUBITAK in 2004. Dr. Karasan received a fellowship from NATO Science Scholarship Program for overseas studies in 1991-94. Dr. Karasan is currently the Bilkent team leader of the FP6-IST Network of Excellence (NoE) project e-Photon/ONe and he also participates in FP6-IST NoE Newcom. His current research interests are in the application of optimization and performance analysis tools for the design, engineering and analysis of optical networks and wireless ad hoc/sensor networks.

Kaan Dogan received his B.S. and M.S. degrees from Electrical and Electronics Engineering Department, Bilkent University, Turkey, in 2003 and 2006 , respectively. He is currently on military duty for the Turkish armed forces. 